\title{
Niacin protects against UVB radiation-induced apoptosis in cultured human skin keratinocytes
}

\author{
FUQUAN LIN, WEN XU, CUIPING GUAN, MIAONI ZHOU, WEISONG HONG, \\ LIFANG FU, DONGYIN LIU and AIE XU
}

\author{
Department of Dermatology, Third People's Hospital of Hangzhou, Hangzhou 310009, P.R. China
}

Received November 10, 2011; Accepted December 23, 2011

DOI: $10.3892 / \mathrm{ijmm} .2012 .886$

\begin{abstract}
Niacin and its related derivatives have been shown to have effects on cellular activities. However, the molecular mechanism of its reduced immunosuppressive effects and photoprotective effects remains unclear. In this study, we investigated the molecular mechanism of the photoprotective effect of niacin in ultraviolet (UV)-irradiated human skin keratinocytes (HaCaT cells). We found that niacin effectively suppressed the UV-induced cell death and cell apoptosis of HaCaT cells. Existing data have shown that AKT activation is involved in the cell survival process. Yet, the potential mechanism of niacin in protection against UV-induced skin damage has thus far not fully been eluvidated. We observed that niacin pretreatment enhances UV induced activation of AKT (Ser473 phosphorylation) as well as that of the downstream signal mTOR (S6 and 4E-BP1 phosphorylation). The PI3K/AKT inhibitor, LY294002, and the mTOR inhibitor, rapamycin, largely neutralized the protective effects of niacin, suggesting that AKT and downstream signaling mTOR/S6 activation are necessary for the niacin-induced protective effects against UV-induced cell death and cell apoptosis. Collectively, our data suggest that niacin may be utilized to prevent UV-induced skin damage and provide a novel mechanism of its photoprotective effects against the UV radiation of sunlight by modulating both AKT and downstream mTOR signaling pathways.
\end{abstract}

\section{Introduction}

Niacin has long been used in the treatment of high cholesterol, coronary heart disease, the skin disease Pellagra and age-related macular degeneration (AMD) $(1,2)$. Niacin and its derivative, niacinamide, have been widely applied in skin care products, including moisturizers, anti-aging products, and rosacea treatments (3). It has been well-accepted that niacin has protective effects against skin photodamage induced

Correspondence to: $\mathrm{Dr}$ Aie Xu, Department of Dermatology, Third People's Hospital of Hangzhou, Hangzhou 310009, P.R. China E-mail: xuaiehz@msn.com

Key words: niacin, UVB, AKT, apoptosis by ultraviolet (UV)-radiation. A niacin derivative, myristyl nicotinate $(\mathrm{MN})$, may enhance epidermal differentiation and barrier function in skin against photodamage, suggesting that niacin delivery may protect against UV skin damage and may be used in treating skin barrier impairment (4).

Niacin has the potential to influence cellular processes including DNA repair, genomic stability, the immune system, stress responses, signaling, transcription, apoptosis, metabolism, differentiation, chromatin structure and life span. In addition to its well-known redox functions in energy metabolism, niacin and its derivatives in the form of NAD and NADP, are required for the synthesis of cyclic ADP-ribose and NAADP, which are two major mediators of intracellular calcium signaling pathways (5).

A previous study (6) showed that niacin-deficient $\mathrm{HaCaT}$ cells with low NAD status develop a decreased growth rate due to an increase in apoptotic cells and an arrest in the G2/M cell cycle phase accompanied with accumulation of reactive oxygen species and increased DNA damage. This model of niacin deficiency has also allowed the identification of NAD-dependent signaling events critical in early skin carcinogenesis. Niacin deficiency in humans would cause sun sensitivity in the skin, indicative of deficiencies in responding to UV damage.

Furthermore, the identification of the nicotinic acid receptor in human skin keratinocytes provides a further link to niacin's role as a potential skin cancer prevention agent and supports the role of the nicotinic acid receptor as a potential target for skin cancer prevention agents (7). While the exact mechanism through which niacin serves as a modulator agent for a critical resistance factor to prevent from UV-induced skin damage is still unknown. Cellular energy loss (8) and AMPK, AKT and eNOS activation (9) have been suggested as some of the niacin protective mechanisms.

UV is divided into UVC (200-280 nm), UVB (280-320 nm) and UVA (320-400 $\mathrm{nm})$. UVB is of environmental significance, penetrating into the papillary area of the dermis and inducing DNA damages to the residing dendritic cells (DC) (10-12), as well as keratinocytes. These cells are perturbed both phenotypically and functionally, undergoing apoptosis upon UVB-radiation. Apoptosis can be induced in the region that suffers the greatest exposure and cells surrounding this area would also be partially damaged $(10,12)$.

Apoptosis is the process of programmed cell death, which involves a series of morphological changes, including cell 
detachment, cell shrinkage, mitochondria leakage, chromatin condensation, and DNA fragmentation. This process is controlled by the balance between pro-apoptotic and anti-apoptotic signaling pathways (13). The PI3K/AKT and JNK phosphorylation cascades act as two important regulators, and will have an anti-apoptotic role in a variety of tissue culture models against stresses such as UV irradiation, matrix detachment, cell cycle disturbance, and DNA damages $(14,15)$.

Previous studies in dendritic cells as well as keratinocytes have demonstrated that the cellular response to UV is composed of transactivation of cell surface growth factors, such as EGFR, and their downstream signal transduction machinery such as MAPK and PI3K/AKT (12,16-19). UV activates all three MAPK families including JNK, p38 and ERK in human keratinocytes and skin dendritic cells (12,20-25). While MAPK (JNK and p38) is responsible for UV-induced cell apoptosis, other cellular signals such as AKT serve as survival signals to fight against UV-induced widespread cell death $(17,19,26,27)$. Activated AKT relays its survival signals in a number of ways including phosphorylation and inactivation of the pro-apoptotic BH3 protein BAD (28), members of the Forkhead family of transcription factors (29) and Mdm2, the negative regulator of p53 (30). However, the possible role of mTORC1 (mTOR complex 1), another important downstream target of AKT, in UV-induced cell death is not fully studied.

It is possible that niacin affects the cell status by protecting against UV-induced apoptosis and death. To determine the mechanism of niacin in $\mathrm{HaCaT}$ cells under $\mathrm{UV}$, we measured the level of AKT and MAPK after UV irradiation. In this study, we demonstrate that UV radiation induces both AKT and MAPK activation. AKT and TSC2 are required for UV-induced mTOR/S6 activation (S6K and 4E-BP1 phosphorylation). Niacin pretreatment protects against $\mathrm{UV}$-induced cell death and apoptosis in keratinocytes (HaCaT cells) by enhancing AKT/mTOR and S6 activation. Niacin may have a functional role in the pro-survival mechanism through activating the AKT/mTOR/S6 signaling pathway.

\section{Materials and methods}

Materials. Niacin (nicotinic acid) and DMSO were obtained from Sigma (St. Louis, MO, USA). Monoclonal mouse anti- $\beta$ actin, goat anti-rabbit IgG-HRP and goat anti-mouse IgG-HRP antibody were purchased from Santa Cruz Biotechnology, Inc. (Santa Cruz, CA, USA). The Annexin V-FITC kit was purchased from BD Biosciences (San Jose, CA, USA). The JC-1 probe cell apoptosis detection kit was provided by the Beyotime Institute of Biotechnology (Haimen, China). The p-EGFR (Tyr1068) antibody, and the p-AKT (Ser473), p-AKT (Thr308), p-S6K (Thr389), p-4E-BP1 (Ser65), p-S6 (S235/236), p-mTOR (Ser2448), p-p38 (Thr180/Tyr182), p-JNK(Thr183/Tyr185), p-ERK1/2 (Thr202/Tyr 204), and p-TSC2 (Ser 1462) antibodies, the AKT inhibitor LY294002, the mTOR inhibitor rapamycin, the Tuberin/TSC2 siRNA, and the p38 MAPK, JNK and the AKT1/2 antibodies were all from Cell Signaling Technology (Beverly, MA, USA). The EGFR inhibitor, PD153035 was purchased from Invitrogen (Carlsbad, CA, USA).

Cell culture, transfection and UV treatment. The spontaneously immortalized human keratinocytes (HaCaT cell line) were cultured at $37^{\circ} \mathrm{C}$ in RPMI-1640 supplemented with $10 \%$ fetal bovine serum and $100 \mathrm{U} / \mathrm{ml}$ of penicillin/streptomycin as previously reported $(31,32)$. The procedures for UV irradiation were similar to those described previously. For UV irradiation experiments, the cells were starved for 1 day and then washed twice with phosphate-buffered saline (PBS) before being exposed to UV irradiation using a germicidal lamp (emission wave, 290-320 nm; radiation intensity, $1.35 \mathrm{~mW} / \mathrm{cm}^{2}$ ) SS-04B Sigma High-Tech Co., Ltd. (Shanghai, China). Doses of UV-irradiation were calibrated with a UVX radiometer also from Sigma High-Tech Co., Ltd. For siRNA transfection, cells were plated at a density of $3 \times 10^{6}$ cells per $100-\mathrm{mm}$ culture dish, incubated overnight, and then transfected with the indicated expression vectors, using Lipofectamine 2000 (Invitrogen, USA).

Cell viability assay (MTT assay). Cell viability was measured by the 3-[4,5-dimethylthylthiazol-2-yl]-2,5 diphenyl tetrazolium bromide (MTT) method (31). Briefly, cells were seeded in 96-well plates at a density of $1 \times 10^{4}$ cells/well. Different seeding densities were optimized at the beginning of the experiments. After incubation for $24 \mathrm{~h}$, cells were exposed to fresh medium containing reagents at $37^{\circ} \mathrm{C}$. After incubation for up to $24 \mathrm{~h}, 10 \mu \mathrm{l}$ of MTT tetrazolium salt (Sigma) dissolved in Hank's balanced solution at a concentration of $5 \mathrm{mg} / \mathrm{ml}$ was added to each well and incubated in $\mathrm{CO}_{2}$ incubator for $4 \mathrm{~h}$. The DMSO $(10 \mu \mathrm{l})$ was added to dissolve formazan crystals and then lysed on a shaker for $15 \mathrm{~min}$. The absorbance value at $490 \mathrm{~nm}$ was measured with a microplate spectrophotometer (SoftMax ${ }^{\circledR}$ Pro5, Molecular Devices, USA).

Western blot analysis. As described previously (33), post treatment, cells were collected and lysed in RIPA buffer (10 mM NaPO, pH 7.4/300 mM NaCl/0.1\% SDS/1\% Nonidet P-40/1\% deoxycholic acid/2 mM EDTA) with protease inhibitors (Pierce, USA). Aliquots of 30-40 $\mu \mathrm{g}$ of protein from each sample (treated as indicated in the legends) were separated by $10 \%$ SDS-polyacrylamide gel electrophoresis (SDS-PAGE) and transferred onto a polyvinylidenedifluoride (PVDF) membrane (Millipore, Bedford, MA). After blocking with 5\% BSA (Beyotime) for $1 \mathrm{~h}$, membranes were incubated with specific antibodies overnight at $4^{\circ} \mathrm{C}$ followed by incubation with secondary antibodies (HRP-conjugated anti-rabbit or anti-mouse IgG at the appropriate dilutions) for $45 \mathrm{~min}$ to $1 \mathrm{~h}$ at room temperature. Immunoblotted membranes were developed using the ECL western blotting system (Thermo Scientific Biotechnology, USA). Quantification of appropriate bands on western blots was performed using the quantification software of the digital imaging system (ChemiDoc ${ }^{\mathrm{TM}} \mathrm{XRS}^{+}$, Bio-Rad, USA).

Confocal laser scanning microscope (CLSM). As previously described (33), all cells were fixed with $4 \%$ formaldehyde in PBS for 30 min. Fixed cells were blocked with $10 \%$ BSA in PBS for $30 \mathrm{~min}$ at room temperature. Anti-p-AKT and antip-S6 polyclonal antibodies were used at $1 \mu \mathrm{g} / 100 \mu \mathrm{l}$ in buffer (0.5\% BSA in PBS) and incubated with the coverslip for $1 \mathrm{~h}$. The antibodies were detected using appropriate secondary antibodies (goat anti-mouse or goat anti-rabbit; Beyotime, China) conjugated to $\mathrm{Cy} 3$ or FITC at 1:200. The processed slides were observed with a Confocal Laser Scanning Microscope (TCS SP2, Leica, Germany). 


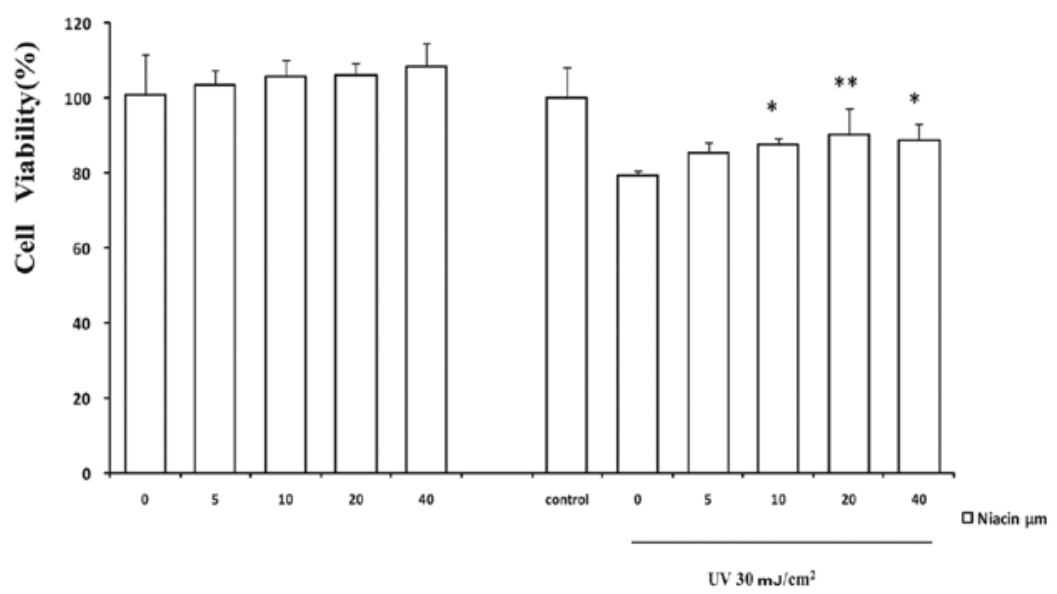

Figure 1. Niacin protects against UVB induced cell death in HaCaT cells. HaCaT cells were pretreated with niacin $(0,5,10,20$ and $40 \mu \mathrm{M})$ for $1 \mathrm{~h}$, followed by $30 \mathrm{~mJ} / \mathrm{cm}^{2} \mathrm{UV}$ radiation. After incubation at $37^{\circ} \mathrm{C}$ for $24 \mathrm{~h}$, cell viability was detected by the MTT assay. The values in the figure are expressed as the means \pm standard deviation (SD). ${ }^{*} \mathrm{P}<0.05,{ }^{* *} \mathrm{P}<0.01$ vs. the UV-treated niacin-free group. All experiments were repeated at least three times and similar results were obtained.

Annexin $V$ and propidium iodide (PI) staining. A cell apoptosis detection kit was used for Annexin V and PI staining. $\mathrm{HaCaT}$ cells were cultured in 35-mm dishes and were exposed to different treatments. Cells were harvested after up to $24 \mathrm{~h}$ incubation and washed in PBS twice. Cells were re-suspended in the binding buffer, and then the fluorescein-conjugated Annexin V and PI reagent were added to cell suspensions. After incubation in the dark for $15 \mathrm{~min}$ the percentage of apoptotic cells and necrotic cells were assessed by a flow cytometer (FACSCalibur, BD Biosciences, USA).

Mitochondrial membrane potential assay. The JC-1 probe was used to measure mitochondrial depolarization. Briefly, cells were cultured in 6-well plates up to $\sim 50 \%$ confluency, and after the indicated treatments cells were incubated with $1 \mathrm{ml}$ of JC- 1 staining solution $(5 \mu \mathrm{g} / \mathrm{ml})$ at $37^{\circ} \mathrm{C}$ for $20 \mathrm{~min}$ and rinsed twice with PBS. Mitochondrial membrane potentials were monitored by determining the relative amounts of dual emissions from mitochondrial JC-1 monomers or aggregates using an Olympus fluorescent microscope under Argon-ion $488 \mathrm{~nm}$ laser excitation. Mitochondrial depolarization is indicated by an increase in the green/red fluorescence intensity.

Statistical analysis. The values in the figures are expressed as the means \pm standard deviation (SD). All experiments were repeated at least three times. Experimental groups were compared by one-way ANOVA analysis of SPSS 16.0. Mean differences were considered significant $(\mathrm{P}<0.05)$ and highly significant $(\mathrm{P}<0.01)$.

\section{Results}

Niacin protects against $U V$-induced cell death and apoptosis in keratinocytes ( $\mathrm{HaCaT}$ cells). By using the cell viability MTT assay, first we investigated the possible protective effects of niacin against UV-induced cell death. As indicated in Fig. 1, cells were pretreated with a dose-dependent course of niacin $(0$, $5,10,20$ and $40 \mu \mathrm{M})$, after with or without $\mathrm{UV}\left(30 \mathrm{~mJ} / \mathrm{cm}^{2}\right)$ as indicated in similar studies. There was no significant dosage effect with up to $40 \mu \mathrm{M}$ of niacin in the control groups. As expected, UV treatment caused a marked decrease in cell viability. The viable cells dropped to $79.35 \pm 1.21 \%(n=4)$ of the control within $24 \mathrm{~h}$ of $30 \mathrm{~mJ} / \mathrm{cm}^{2} \mathrm{UV}$ radiation. In the presence of niacin, the cell viability was markedly recovered to $90.21 \pm 7.09 \%(\mathrm{P}<0.01)$ at $20 \mu \mathrm{M}$ of niacin in comparison to the UV-treated and niacin-free group. Significant improvement of cell viability occurred with a graded increase in niacin concentrations from 5 to $20 \mu \mathrm{M}$, and showed a declining trend at $40 \mu \mathrm{M}$ with UV treatment. Next we assessed whether niacin can inhibit UV-induced cell apoptosis. Data in Fig. 2C clearly demonstrate that $20 \mu \mathrm{M}$ with niacin pretreatment diminishes UV induced cell apoptosis in HaCaT cells as evidenced by the Annexin V and PI staining assay (Fig. 3). Normal cells stained with the JC-1 probe emitted mitochondrial red fluorescence and limited green fluorescence. Aggregated JC-1 has a high mitochondrial membrane potential and emits red fluorescence but will turn green after normal mitochondria is dispersed to the monomeric form. Pretreatment with $20 \mu \mathrm{M}$ niacin against UV dramatically decreased the green/red fluorescence ratio $(\mathrm{P}<0.01)$ in comparison with UV only, and the apoptotic cells dropped from $26.10 \pm 4.57 \%$ to $18.92 \pm 3.26 \%(\mathrm{P}<0.01)$.

$A K T, T S C 2$ and $m$ TOR are required for $U V$-induced AKT cascade activation. We next examined whether the AKT cascade is activated upon UV radiation. The results showed that UV radiation activates the EGFR/AKT/mTOR pathway in a time- and dose-dependent manner. UV radiation $(30 \mathrm{~mJ} /$ $\mathrm{cm}^{2}$ ) activates AKT at the highest level. The peaks of EGFR/ AKT/mTOR activation take place between 30 and $60 \mathrm{~min}$ at the UV dose of $30 \mathrm{~mJ} / \mathrm{cm}^{2}$ (Fig. 2A and B). Previous studies have demonstrated the key role of AKT in mTOR activation in response to growth factors (32). To investigate the role of AKT in UV-induced mTOR/S6 activation, AKT cascade inhibitors were used. The results show that UV does not induce AKT/ mTOR/S6 activation in cells treated with the EGFR inhibitor, PD153035, or the AKT inhibitor, LY294002 (Fig. 2D and E), suggesting that both EGFR and AKT are required for UV-induced mTOR/S6 activation. Previous studies have also shown that the regulation of S6K and 4E-BP1 by AKT occurs primarily through the phosphorylation and inactivation of 
A

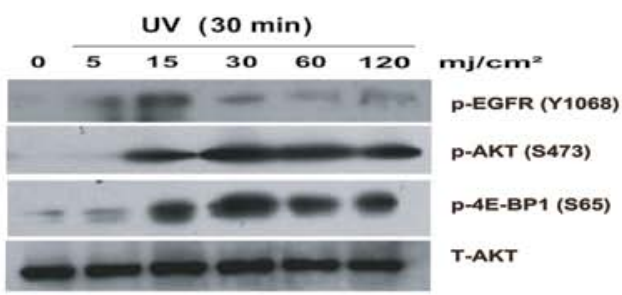

C

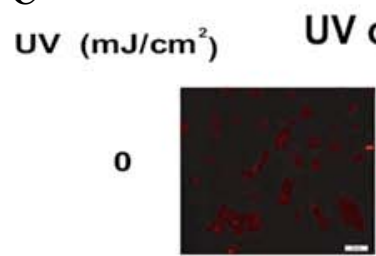

15

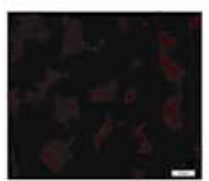

30

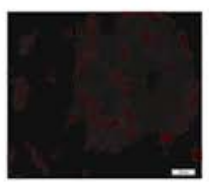

60

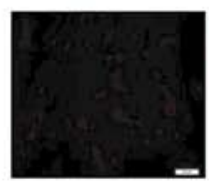

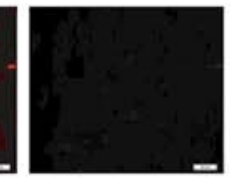
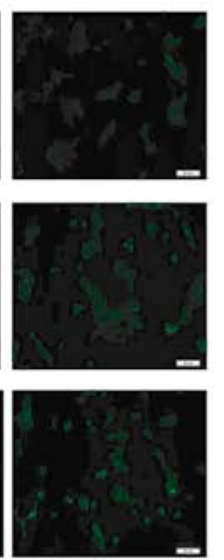
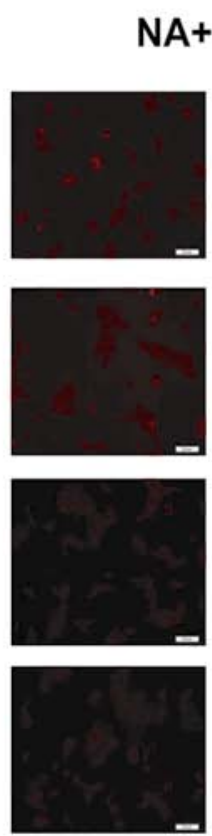

B

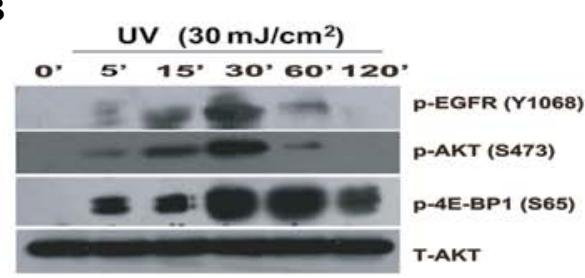
UV $\left(30 \mathrm{~mJ} / \mathrm{cm}^{2}\right)$

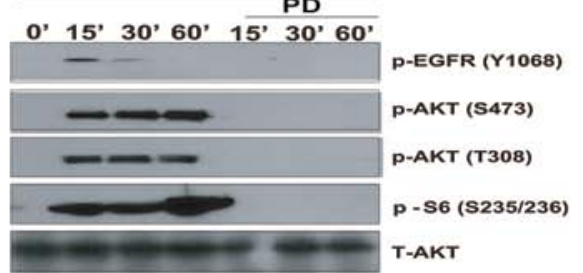

$\mathbf{E}$ UV $\left(30 \mathrm{~mJ} / \mathrm{cm}^{2}\right)$
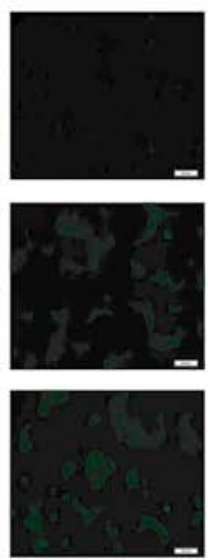

E Rapa LY
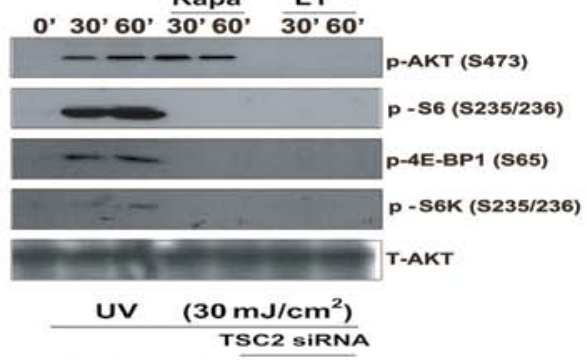

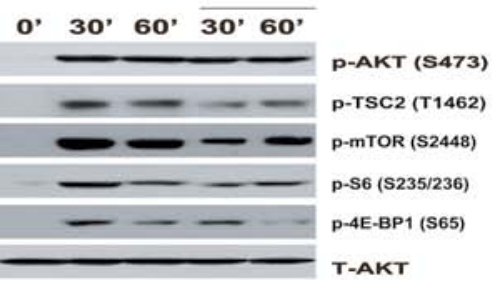

Figure 2. AKT, TSC2, mTOR and S6 are required for UV-induced AKT cascade activation. (A and B) HaCaT cells were exposed to different dosages of UV radiation $\left(0,5,15,30,60,120 \mathrm{~mJ} / \mathrm{cm}^{2}\right)$ for $30 \mathrm{~min}$ and for different durations of $30 \mathrm{~mJ} / \mathrm{cm}^{2} \mathrm{UV}$ respectively. p-EGFR (Tyr1068), p-AKT (Ser473), p-4E-BP1 (Ser65) were detected by western blotting. (C) HaCaT cells were pretreated with $20 \mu \mathrm{M}$ niacin, followed by UV radiation $\left(0,15,30,60 \mathrm{~mJ} / \mathrm{cm}^{2}\right)$, then stained with the JC-1 probe and imaged by fluorescent microscope. The individual red and green average fluorescence intensities are expressed as the ratio of green to red fluorescence. An increase on the fluorescence green/red ratio indicates a shift increase in mitochondrial depolarization as an early apoptosis label. HaCaT cells were pre-treated with (D) the EGFR inhibitor, PD153035 (PD, $1 \mu \mathrm{M}$ ); (E) with the PI3K/AKT inhibitor LY294002 (LY, $10 \mu \mathrm{M})$ and mTOR inhibitor rapamycin (Rapa, $100 \mathrm{nM}$ ), for $1 \mathrm{~h}$, with $\mathrm{UV}\left(30 \mathrm{~mJ} / \mathrm{cm}^{2}\right)$ radiation and cultured for 15, 30 and $60 \mathrm{~min}$. (F) Cells were transfected with Tuberin/TSC2 siRNAII $(100 \mathrm{nM})$ for $48 \mathrm{~h}$ prior to UV radiation $\left(30 \mathrm{~mJ} / \mathrm{cm}^{2}\right.$ ), for 30 or $60 \mathrm{~min}$. p-EGFR (Tyr1068), p-AKT (Ser473), p-AKT (Thr308), p-TSC2 (Thr1462), p-mTOR (Ser2448), p-S6K (Thr389), p-S6 (Ser235/236), p-4E-BP1 (Ser65) and total AKT antibody were used by western blotting. All experiments were repeated at least three times and similar results were obtained.

TSC2 at Tyr1462 (34), leading to increased phosphorylation of mTOR and downstream S6K and 4E-BP1. We next examined the role of TSC2 in S6K activation upon UV radiation. As shown in Fig. 2F, UV-induced mTOR/S6 activation is modulated by TSC 2 . Transfection with TSC 2 siRNA specifically silenced TSC 2 by approximately $60 \%$, and the downstream mTOR and S6 signals were partially inhibited (Fig. 2F).

Niacin, in contrast to AKT cascade inhibitors, affects $U V$-induced apoptosis in HaCaT cells. As demonstrated, niacin protects against UV-induced cell death and cell apoptosis. The AKT cascade activation is induced by UV radiation. Taken together, we conclude that the AKT cascade signal activation serves as a critical upstream signal against UV, serving as a pro-survival signal against UV-induced cell apoptosis. To determine whether the AKT signaling pathway is involved in the pro-survival and anti-apoptotic effects of niacin, cells were treated with AKT cascade kinase inhibitors independently, such as the EGFR inhibitor, PD153035, the AKT inhibitor, LY294002 and the mTOR inhibitor, rapamycin. As previously shown, treatment with PD153035, LY294002 and rapamycin resulted in the increase of UV-induced apoptosis. Living cells were decreased successively from $93.21 \pm 2.74 \%$ to $80.08 \pm 3.20 \%$ while the percentages of apoptotic cells were successively increased from $6.35 \pm 0.32 \%$ in the control to $18.39 \pm 0.55 \%(\mathrm{P}<0.01)$ in the rapamycin group. Independent treatment with LY294002 and rapamycin seemed to affect cells efficiently. The contribution of the AKT cascade signal activation after UV was further detected by pretreating cells with inhibitors before UV exposure. Treatment with LY294002 and rapamycin were found to have less influence on the UV-induced loss of cell viability in the cells in comparison with PD98059. However, LY294002 has a more critical role in the UV effect. The UV-induced apoptosis 


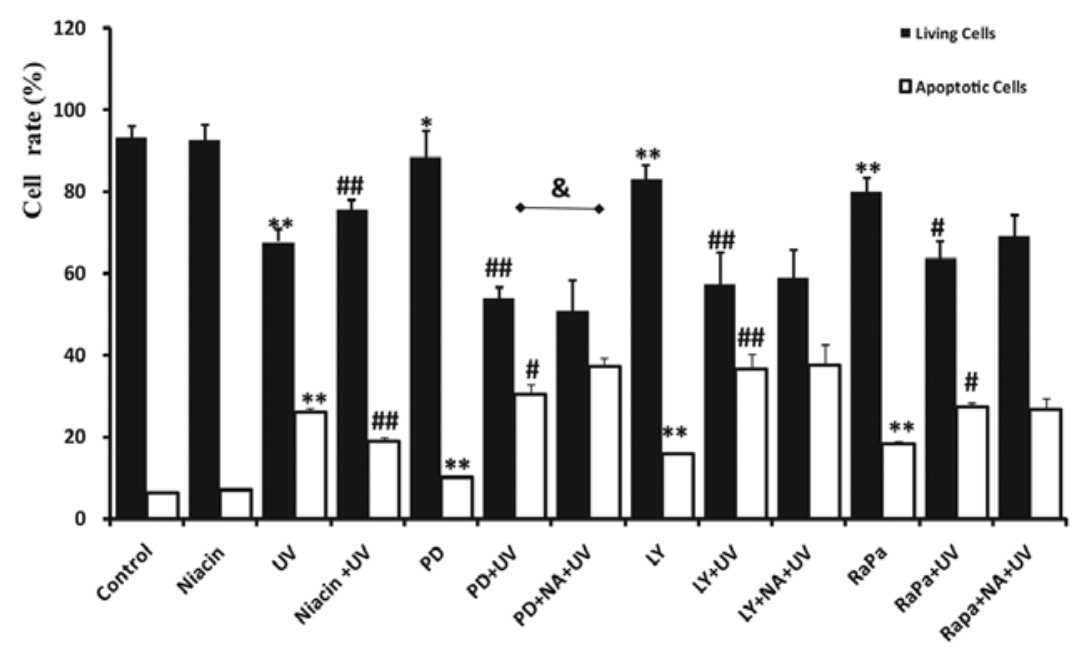

Figure 3. Niacin protects HaCaT cells from UV-induced apoptosis cooperated with different AKT cascade inhibitors. HaCaTs were pre-treated with $20 \mu \mathrm{M}$ niacin (NA) for $30 \mathrm{~min}$, and then incubated with different inhibitors for $30 \mathrm{~min}$ until $30 \mathrm{~mJ} / \mathrm{cm}^{2}$ of UV radiation. PD, LY, RaPa are abbreviations for PD153035, LY294002, and rapamycin respectively. Twenty-four hours post-treatment the number and percentage of apoptotic and necrotic cells were quantified by FACS after cells were washed and stained with Annexin V and PI. Apoptotic cells were determined by counting the percentage of early apoptotic cells Annexin V(+), $\mathrm{PI}(-)$ cells plus the percentage of late apoptotic Annexin V(+), PI(+) cells. Normal living cells are presented as Annexin V(-), PI(-), respectively. The values in the figure are shown as mean $\pm \mathrm{SD}$ of at least three separate experiments. ${ }^{*} \mathrm{P}<0.05,{ }^{* *} \mathrm{P}<0.01$ vs. the control group; ${ }^{*} \mathrm{P}<0.05,{ }^{\# /} \mathrm{P}<0.01$ vs. the $\mathrm{UV}$ only group; and ${ }^{\circledR} \mathrm{P}<0.05$ when comparing the $\mathrm{PD}+\mathrm{UV}$ and $\mathrm{PD}+\mathrm{NA}+\mathrm{UV}$ group.

ratio was $36.71 \pm 3.67 \%$ compared to others. Last, we treated niacin-treated cells with inhibitors prior to UV. This led to an insignificant decrease of UV-induced cell apoptosis $(\mathrm{P}>0.05)$ except for PD98059 which increaed apoptotic cells $(\mathrm{P}<0.05)$.

Niacin protects against $U V$-induced apoptosis via enhancement of AKT and mTORC/S6 activation. We have shown that niacin pretreatment protects against UV-induced cell death and apoptosis in keratinocytes (HaCaT cells) (Figs. 1 and 2). AKT and the downstream signal mTOR/S6 are important for the protective effect of niacin, so next we examined whether niacin is able to induce AKT/mTOR/S6 activation in $\mathrm{HaCaT}$ cells. The results showed that niacin strongly induces AKT and the downstream mTOR/S6 activation at $30 \mathrm{~min}$ (Fig. 4A). Furthermore, niacin induces little activation of MAPK (ERK and JNK) compared to the UV group which strongly activates MAPK (p38) (Fig. 4C). We also demonstrated that UV-induced AKT/ mTOR/S6 activation serves as a prosurvival signal (Fig. 2). Next we examined the possible role of niacin on UV induced AKT/mTOR/S6 activation. As shown in Fig. 4B, niacin pretreatment largely enhances UV-induced AKT/mTOR/S6 activation in HaCaT cells. The AKT inhibitor, LY294002 largely neutralizes the protective effects of niacin (Fig. 4E) and shows a low mitochondrial membrane potential, indicating that AKT activation is necessary for niacin's protective effects against UV-induced cell apoptosis.

\section{Discussion}

Despite previous research showing that niacin reduces the immunosuppressive effects of UV both in vivo and in vitro (35), at least in part by providing energy repletion to UV-irradiated cells for it can normalize subsets of apoptosis and cellular energy loss effects $(8,36)$, the precise molecular mechanisms underlying the cytoprotective and anti-apoptotic effects remain largely unknown. Here, we showed that treatment with niacin led to efficient suppression of UV-induced cell death and apoptosis in keratinocytes (Figs. 1 and 2). We also found that the treatment of niacin could recover the changed mitochondrial membrane potential. These data suggest that niacin can protect against UV-induced mitochondrial dysfunction (Fig. 2). The fact that niacin protects the cells from apoptotic cell damage appears to be applicable to the protection from UV irradiation of keratinocytes, which are the primary cell type in the epidermis and play a key role in the body's initial line of defense.

To understand the molecular mechanism of the antiapoptotic effect of niacin in UV-treated keratinocytes, first we analyzed several signaling pathways related to UV-induced apoptosis in $\mathrm{HaCaT}$ cells. We found that the short time-dependent regulation of the AKT cascade and MAPK feedback activation is involved in $\mathrm{HaCaT}$ cells after $\mathrm{UV}$ exposure. AKT is well known to be differentially activated depending on the type of extracellular stimuli. As suggested in previous studies, MAPK/JNK/p38 activation may be essential for UV-induced apoptosis, but the activation of AKT, mTOR, S6 can be the pro-surviving signals against UV-induced apoptosis. Some plant flavonoids with anti-apoptotic properties activate the MAPK/AKT/NF-кB pathway (37-39). We found that niacin pretreatment largely enhances UV-induced AKT/mTOR/S6 activation in HaCaT cells (Fig. 4). Therefore, the PI3K/AKT cascade is considered as the canonical pathway involved in the protection of UV-induced apoptosis followed by EGFR kinase activation. As indicated in the Fig. 3 apoptosis assay, EGFR could relatively strengthen the damage of UV; AKT and mTOR inhibitors could largely neutralize the protective effects of niacin against UV, suggesting that AKT signaling activation is necessary for niacin's protective effects against UV-induced cell death and apoptosis.

In addition to stimulating cell growth, mTOR also promotes cell survival. eIF4E serves as an important downstream 
A

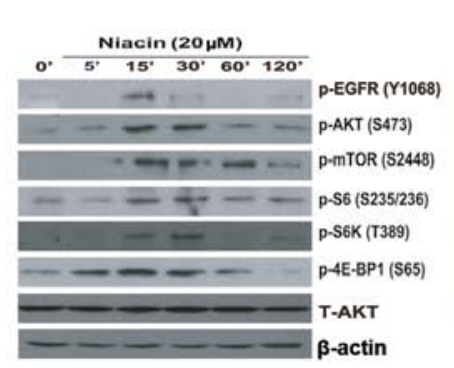

B

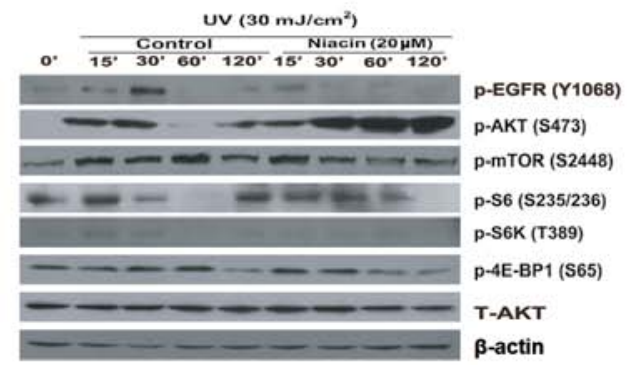

C

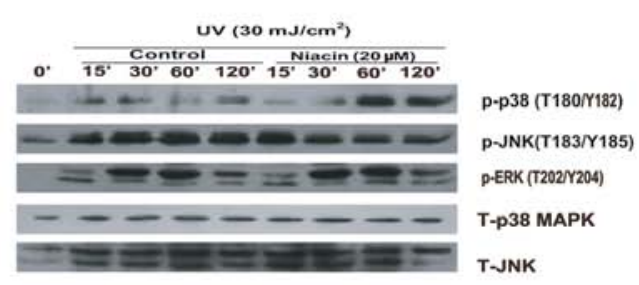

$\mathbf{D}$

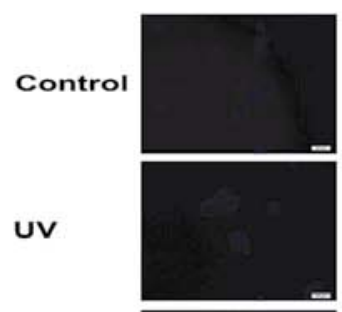

Niacin

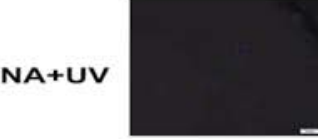

p-AKT (S473)
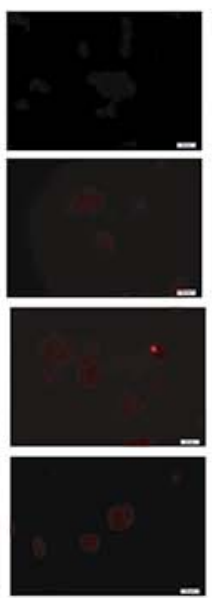

p-S6 (S235/236)
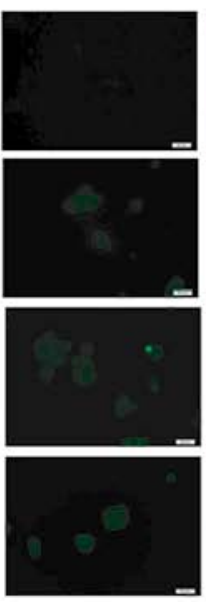

p-AKT Merge
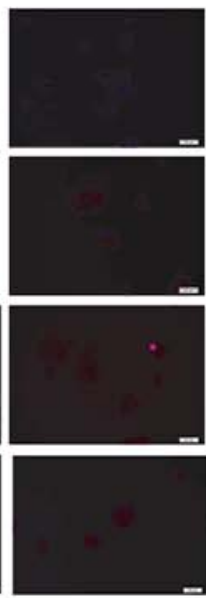

p-S6 Merge
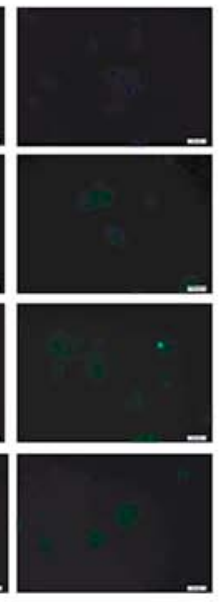

$\mathbf{E}$
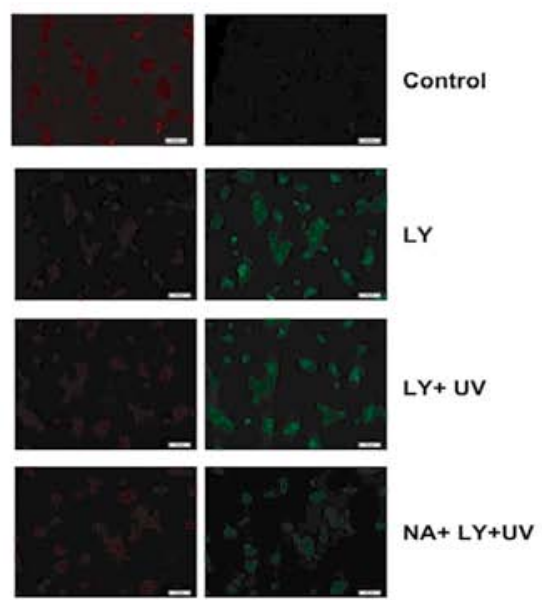

Figure 4. Niacin protects against UV-induced apoptosis via enhancement of AKT/mTOR/S6 activation. (A) HaCaT cells were treated with niacin (NA, $20 \mu \mathrm{M}$ ) and cultured for 5, 15, 30, 60 and 120 min. p-EGFR (Tyr1068), p-AKT (Ser473), p-mTOR (Ser2448), p-S6K (Thr389), p-S6 (Ser235/236), p-4E-BP1 (Ser65), total AKT and $\beta$-actin antibody were used for western blotting. (B and C) HaCaT cells were pretreated with or without niacin (20 $\mu \mathrm{M})$ for $1 \mathrm{~h}$, followed by UV $\left(30 \mathrm{~mJ} / \mathrm{cm}^{2}\right.$ ) radiation and cultured for the indicated time, p-EGFR (Tyr1068), p-AKT (Ser473), p-mTOR (Ser2448), p-S6K (Thr389), p-S6 (Ser235/236), p-4E-BP1 (Ser65), p-p38 (Thr180/Tyr182), p-JNK (Thr183/Tyr185), p-ERK1/2 (Thr202/Tyr204), p38 MAPK, JNK total AKT and $\beta$-actin were detected by western blotting. (D) HaCaT cells were pretreated with niacin $(20 \mu \mathrm{M})$ for $1 \mathrm{~h}$, followed by UV radiation $\left(30 \mathrm{~mJ} / \mathrm{cm}^{2}\right)$ and cultured for $1 \mathrm{~h}$, p-AKT (Ser473) and p-S6 (Ser235/236) were detected by confocal microscopy. (E) HaCaT cells were pre-treated with niacin and LY294002 separately or in combination, after UV $\left(30 \mathrm{~mJ} / \mathrm{cm}^{2}\right)$ radiation; mitochondrial depolarization was observed the same way in Fig. $2 \mathrm{C}$.

effectors of mTOR in the control of cell survival through the 4E-BP1 (40-46). The translation and expression of mRNAs encoding a few major anti-apoptotic proteins including (XIAP, c-IAP1, Bcl-XL and BCl-2) are mTORC1 and cap-dependent (47). S6K1 is another mTOR target that plays a role in the apoptosis resistance of cancer cells (48-50). Previous studies (51) together with our current work demonstrate that mTOR activation serves as a pro-survival UV-induced cell apoptosis signal (Figs. 2 and 4). AKT, by phosphorylation and inhibition of TSC2, serves as the upstream signal for UV-induced mTORC1 activation (Fig. 2). Importantly, UV-induced TSC2 and S6K phosphorylation were almost abolished by pharmacological inhibitors of AKT (Fig. 2). Taken together, we conclude that AKT by phosphorylating and inhibiting TSC2, acts as an upstream signal for UV-induced mTOR activation, and the latter serves as a pro-survival signal against UV-induced cell death and apoptosis.

Although the PI3K/AKT and its downstream substrate mTOR (S6K and 4E-BP1 phosphorylation, rapamycin sensitive) pathway are well-established, the identity of the kinase responsible for phosphorylating AKT at Ser473 remains elusive until in recent years, when it was revealed to be
mTORC2 (46,52-54). mTORC2 is a complex of mLST8, rictor (rapamycin-insensitive companion of mTOR), and mSin 1 with mTOR (46,52-56). Aside from AKT hydrophobic motif phosphorylation, mTORC2 has also been implicated in the phosphorylation of the AGC kinase, PKC $\alpha(46,57)$, the regulation of actin cytoskeleton reorganization (46) and development of prostate cancer in PTEN deficient mice (58). Ablation of mTORC2 activity impairs the phosphorylation of only a subset of AKT targets including FoxO1/3a, while leaving other AKT targets such as TSC2 and glycogen synthase kinase 3 (GSK-3) as well as the mTORC1 effectors S6K and 4E-BP1 unaffected $(46,57,59)$.

In conclusion, we found for the first time that niacin pretreatment protects against UV-induced cell death and apoptosis by enhancing the pro-survival pathways including AKT, mTOR and S6 in skin keratinocytes (HaCaT cells). Oral and external niacin and nicotinamide are both safe and inexpensive and appear to be promising chemopreventive supplements for reducing the mutagenic, immunosuppressive and cell damage effects of sunlight (60). To our knowledge, this is also the first study providing a molecular mechanism to support that niacin can be utilized as a skin photodamage protective agent. 


\section{Acknowledgements}

This research was supported by a grant from the National Natural Science Foundation of China (30872280 to Dr Aie Xu).

\section{References}

1. Studer M, Briel M, Leimenstoll B, Glass TR and Bucher HC: Effect of different antilipidemic agents and diets on mortality: a systematic review. Arch Intern Med 165: 725-730, 2005.

2. Metelitsina TI, Grunwald JE, DuPont JC and Ying GS: Effect of niacin on the choroidal circulation of patients with age related macular degeneration. Br J Ophthalmol 88: 1568-1572, 2004.

3. Bissett DL, Oblong JE and Berge CA: Niacinamide: A B vitamin that improves aging facial skin appearance. Dermatol Surg 31: $860-865,2005$.

4. Jacobson EL, Kim H, Kim M, et al: A topical lipophilic niacin derivative increases NAD, epidermal differentiation and barrier function in photodamaged skin. Exp Dermatol 16: 490-499, 2007.

5. Kirkland JB: Niacin and carcinogenesis. Nutr Cancer 46: 110-118, 2003

6. Benavente CA and Jacobson EL: Niacin restriction upregulates NADPH oxidase and reactive oxygen species (ROS) in human keratinocytes. Free Radic Biol Med 44: 527-537, 2008.

7. Benavente CA, Jacobson MK and Jacobson EL: NAD in skin: therapeutic approaches for niacin. Curr Pharm Des 15: 29-38, 2009.

8. Park J, Halliday GM, Surjana D and Damian DL: Nicotinamide prevents ultraviolet radiation-induced cellular energy loss Photochem Photobiol 86: 942-948, 2010.

9. Penumathsa SV, Thirunavukkarasu M, Samuel SM, et al: Niacin bound chromium treatment induces myocardial Glut-4 translocation and caveolar interaction via Akt, AMPK and eNOS phosphorylation in streptozotocin induced diabetic rats after ischemia-reperfusion injury. Biochim Biophys Acta 1792: 39-48, 2009.

10. Meunier L: Ultraviolet light and dendritic cells. Eur J Dermatol 9: 269-275, 1999.

11. Kolgen W, Both $\mathrm{H}$, van Weelden $\mathrm{H}$, et al: Epidermal langerhans cell depletion after artificial ultraviolet B irradiation of human skin in vivo: apoptosis versus migration. J Invest Dermatol 118: $812-817,2002$

12. Nakagawa S, Ohtani T, Mizuashi M, et al: p38 Mitogen-Activated protein kinase mediates dual role of ultraviolet $\mathrm{B}$ radiation in induction of maturation and apoptosis of monocyte-derived dendritic cells. J Invest Dermatol 123: 361-370, 2004.

13. McGowan BS, Ciccimaro EF, Chan TO and Feldman AM: The balance between pro-apoptotic and anti-apoptotic pathways in the failing myocardium. Cardiovasc Toxicol 3: 191-206, 2003.

14. Datta SR, Brunet A and Greenberg ME: Cellular survival: a play in three Akts. Genes Dev 13: 2905-2927, 1999.

15. Burke RE: Inhibition of mitogen-activated protein kinase and stimulation of Akt kinase signaling pathways: two approaches with therapeutic potential in the treatment of neurodegenerative disease. Pharmacol Ther 114: 261-277, 2007.

16. Zhuang L, Wang B, Shinder GA, Shivji GM, Mak TW and Sauder DN: TNF receptor p55 plays a pivotal role in murine keratinocyte apoptosis induced by ultraviolet B irradiation. J Immunol 162: 1440-1447, 1999.

17. Xu Y, Voorhees JJ and Fisher GJ: Epidermal growth factor receptor is a critical mediator of ultraviolet $\mathrm{B}$ irradiation-induced signal transduction in immortalized human keratinocyte $\mathrm{HaCaT}$ cells. Am J Pathol 169: 823-830, 2006.

18. Sachsenmaier C, Radler-Pohl A,Zinck R, Nordheim A, Herrlich P and Rahmsdorf HJ: Involvement of growth factor receptors in the mammalian UVC response. Cell 78: 963-972, 1994.

19. Wan YS, Wang ZQ, Shao Y, Voorhees JJ and Fisher GJ: Ultraviolet irradiation activates PI 3-kinase/AKT survival pathway via EGF receptors in human skin in vivo. Int J Oncol 18: 461-466, 2001

20. Pfundt R, van Vlijmen-Willems I, Bergers M, Wingens M, Cloin W and Schalkwijk J: In situ demonstration of phosphorylated c-jun and p38 MAP kinase in epidermal keratinocytes following ultraviolet B irradiation of human skin. J Pathol 193: 248-255, 2001

21. Chen W, Tang Q, Gonzales MS and Bowden GT: Role of p38 MAP kinases and ERK in mediating ultraviolet-B induced cyclooxygenase-2 gene expression in human keratinocytes. Oncogene 20: 3921-3926, 2001.
22. Bode $\mathrm{AM}$ and Dong Z: Mitogen-activated protein kinase activation in UV-induced signal transduction. Sci STKE 2003: 167, 2003.

23. Papoutsaki M, Moretti F, Lanza M, et al: A p38-dependent pathway regulates DeltaNp63 DNA binding to p53-dependent promoters in UV-induced apoptosis of keratinocytes. Oncogene 24: 6970-6975, 2005.

24. Chouinard N, Valerie K, Rouabhia M and Huot J: UVB-mediated activation of p38 mitogen-activated protein kinase enhances resistance of normal human keratinocytes to apoptosis by stabilizing cytoplasmic p53. Biochem J 365: 133-145, 2002.

25. Zhang QS, Maddock DA, Chen JP, et al: Cytokine-induced p38 activation feedback regulates the prolonged activation of AKT cell survival pathway initiated by reactive oxygen species in response to UV irradiation in human keratinocytes. Int J Oncol 19: 1057-1061, 2001.

26. Wang S and El-Deiry WS: TRAIL and apoptosis induction by TNF-family death receptors. Oncogene 22: 8628-8633, 2003.

27. Li Y, Bi Z, Yan B and Wan Y: UVB radiation induces expression of HIF-1 $\alpha$ and VEGF through the EGFR/PI3K/DEC1 pathway. Int J Mol Med 18: 713-719, 2006.

28. Datta SR, Dudek H, Tao X, et al: Akt phosphorylation of BAD couples survival signals to the cell-intrinsic death machinery. Cell 91: 231-241, 1997.

29. Brunet A, Bonni A, Zigmond MJ, et al: Akt promotes cell survival by phosphorylating and inhibiting a Forkhead transcription factor. Cell 96: 857-868, 1999.

30. Mayo LD and Donner DB: A phosphatidylinositol 3-kinase/Akt pathway promotes translocation of Mdm2 from the cytoplasm to the nucleus. Proc Natl Acad Sci USA 98: 11598-11603, 2001.

31. Cao C, Healey S, Amaral A, et al: ATP-sensitive potassium channel: a novel target for protection against UV-induced human skin cell damage. J Cell Physiol 212: 252-263, 2007.

32. Cao C, Sun Y, Healey S, et al: EGFR-mediated expression of aquaporin-3 is involved in human skin fibroblast migration. Biochem J 400: 225-234, 2006.

33. Guan C, Lin F, Zhou M, et al: The role of VIT1/FBXO11 in the regulation of apoptosis and tyrosinase export from endoplasmic reticulum in cultured melanocytes. Int J Mol Med 26: 57-65, 2010.

34. Dickenson JM, Reeder S, Rees B, Alexander S and Kendall D: Functional expression of adenosine A2A and A3 receptors in the mouse dendritic cell line XS-106. Eur J Pharmacol 474: 43-51, 2003.

35. Sivapirabu G, Yiasemides E, Halliday GM,Park J and Damian DL: Topical nicotinamide modulates cellular energy metabolism and provides broad-spectrum protection against ultraviolet radiationinduced immunosuppression in humans. Br J Dermatol 161: 1357-1364, 2009.

36. Damian DL: Photoprotective effects of nicotinamide. Photochem Photobiol Sci 9: 578-585, 2010.

37. Lee ER, Kim JH, Choi HY, Jeon K and Cho SG: Cytoprotective effect of eriodictyol in UV-irradiated keratinocytes via phosphatase-dependent modulation of both the p38 MAPK and Akt signaling pathways. Cell Physiol Biochem 27: 513-524, 2011.

38. Anggakusuma, Yanti and Hwang JK: Effects of macelignan isolated from Myristica fragrans Houtt. On UVB-induced matrix metalloproteinase-9 and cyclooxygenase- 2 in $\mathrm{HaCaT}$ cells. J Dermatol Sci 57: 114-122, 2010.

39. Svobodova A, Zdarilova A and Vostalova J: Lonicera caerulea and Vaccinium myrtillus fruit polyphenols protect $\mathrm{HaCaT}$ keratinocytes against UVB-induced phototoxic stress and DNA damage. J Dermatol Sci 56: 196-204, 2009.

40. Edinger AL and Thompson CB: Akt maintains cell size and survival by increasing mTOR-dependent nutrient uptake. Mol Biol Cell 13: 2276-2288, 2002.

41. Mungamuri SK, Yang X, Thor AD and Somasundaram K: Survival signaling by Notch1: mammalian target of rapamycin (mTOR)-dependent inhibition of p53. Cancer Res 66: 4715-4724, 2006.

42. Polunovsky VA, Gingras AC, Sonenberg N, et al: Translational control of the antiapoptotic function of Ras. J Biol Chem 275: 24776-24780, 2000.

43. Li S, Sonenberg N, Gingras AC, et al: Translational control of cell fate: availability of phosphorylation sites on translational repressor 4E-BP1 governs its proapoptotic potency. Mol Cell Biol 22: 2853-2861, 2002

44. Liotta LA, Steeg PS and Stetler-Stevenson WG: Cancer metastasis and angiogenesis: an imbalance of positive and negative regulation. Cell 64: 327-336, 1991.

45. Proud CG: The eukaryotic initiation factor 4E-binding proteins and apoptosis. Cell Death Differ 12: 541-546, 2005. 
46. Facchinetti V, Ouyang W, Wei H, et al: The mammalian target of rapamycin complex 2 controls folding and stability of Akt and protein kinase C. EMBO J 27: 1932-1943, 2008.

47. Proud CG: Role of mTOR signalling in the control of translation initiation and elongation by nutrients. Curr Top Microbiol Immunol 279: 215-244, 2004.

48. Wang HQ, Quan T, He T, Franke TF, Voorhees JJ and Fisher GJ: Epidermal growth factor receptor-dependent, NF-kappaBindependent activation of the phosphatidylinositol 3-kinase/Akt pathway inhibits ultraviolet irradiation-induced caspases-3, -8, and -9 in human keratinocytes. J Biol Chem 278: 45737-45745, 2003.

49. Mamane Y, Petroulakis E, LeBacquer O and Sonenberg N: mTOR, translation initiation and cancer. Oncogene 25: 64166422, 2006.

50. Panner A, James CD, Berger MS and Pieper RO: mTOR controls FLIPS translation and TRAIL sensitivity in glioblastoma multiforme cells. Mol Cell Biol 25: 8809-8823, 2005.

51. Cao C, Lu S, Sowa A, et al: Priming with EGFR tyrosine kinase inhibitor and EGF sensitizes ovarian cancer cells to respond to chemotherapeutical drugs. Cancer Lett 266: 249-262, 2008.

52. Sarbassov DD, Guertin DA, Ali SM and Sabatini DM: Phosphorylation and regulation of Akt/PKB by the rictor-mTOR complex. Science 307: 1098-1101, 2005.

53. Hresko RC and Mueckler M: mTOR.RICTOR is the Ser 473 kinase for Akt/protein kinase B in 3T3-L1 adipocytes. J Biol Chem 280: 40406-40416, 2005.
54. Shiota C, Woo JT, Lindner J, Shelton KD and Magnuson MA: Multiallelic disruption of the rictor gene in mice reveals that mTOR complex 2 is essential for fetal growth and viability. Dev Cell 11: 583-589, 2006.

55. Frias MA, Thoreen CC, Jaffe JD, et al: $\mathrm{mSin} 1$ is necessary for $\mathrm{Akt} / \mathrm{PKB}$ phosphorylation, and its isoforms define three distinct mTORC2s. Curr Biol 16: 1865-1870, 2006.

56. Masri J, Bernath A, Martin J, et al: mTORC2 activity is elevated in gliomas and promotes growth and cell motility via overexpression of rictor. Cancer Res 67: 11712-11720, 2007.

57. Guertin DA, Stevens DM, Thoreen CC, et al: Ablation in mice of the mTORC components raptor, rictor, or mLST8 reveals that mTORC2 is required for signaling to Akt-FOXO and PKCalpha, but not S6K1. Dev Cell 11: 859-871, 2006.

58. Guertin DA, Stevens DM, Saitoh M, et al: mTOR complex 2 is required for the development of prostate cancer induced by Pten loss in mice. Cancer Cell 15: 148-159, 2009.

59. Bhaskar PT and Hay N: The two TORCs and Akt. Dev Cell 12: 487-502, 2007.

60. Yiasemides E, Sivapirabu G, Halliday GM, Park J and Damian DL: Oral nicotinamide protects against ultraviolet radiation-induced immunosuppression in humans. Carcinogenesis 30: 101-105, 2009. 